\title{
Early detection of Prostate Cancer Bangladesh Perspective
}

\author{
MA SALAM
}

(J Banagladesh Coll Phys Surg 2014; 32: 89-93)

\section{Introduction:}

Generally the prostate cancer is a slow growing cancer and early detection may allow an effective protocol of treatment to achieve a long life. The goal of screening for prostate cancer is to find it early, in the hope that it can be treated more effectively. Screening refers to testing to find a disease such as cancer in people who do not have symptoms of that disease. Screening can help find cancers at an early stage, when they are more easily cured. The goal of screening is to help people live healthier, longer lives.

\section{What is the rationale for screening?}

Cancer screening has become part of modern medicine. Today the screening for breast, cervical and colorectal cancer is already normal practice in some countries, and will probably become routine in other countries in the future. Screening for prostate, melanoma and lung cancer are subject to ongoing studies ${ }^{1-3}$. Logic behind screening is simple: to detect cancers at an early stage, when they are still curable.

The screening is currently performed using one of the three methods: mass screening (i.e. large scale screening of an entire population), selective screening (i.e. screening of high-risk populations) or opportunistic screening (e.g. incorporated as part of a medical consultation). Diagnostic testing differs from screening as it attempts to identify the disease in the presence of symptoms, while screening is offered to symptom-free individuals ${ }^{2-5}$.

All screening procedure carries a risk of over diagnosis and overtreatment, which should be balanced against the benefits for those in which the cancers are diagnosed at a curative stage. The potential value of PSA for the early detection of prostate cancer was described in the early 1990s, both prostate cancer incidence and

Address of Correspondence: Professor MA Salam, Chairman, Urology and Transplantation Foundation of Bangladesh, Panthapath, Dhaka, Bangladesh, Former Chairman and Professor of Uro Oncology BSMMU.

Received: 1 January, 2013

Accepted: 15 March, 2014 mortality rates have changed profoundly ${ }^{6}$. For example between 1989 and 2003, the age-standardized incidence rate of prostate cancer increased by $48.4 \%$ in The Netherlands (reaching an incidence of 93.2 cases per 100,000 men). Based on rates from 2001 to 2003, 17.1\% of U.S. men born today will be diagnosed with cancer of the prostate at some time during their lifetime. It is now the most frequently diagnosed non-cutaneous cancer, with 225,000 new cases reported each year in Europe alone ${ }^{7}$.

This increase of incidence suggests that this is due to the increased detection of cancers. This is supported by the reports on autopsy studies have revealed that histologic prostate cancer occurs in an even larger proportion of men compared to the screening incidence: up to $55 \%$ of men in their fifties, and $64 \%$ of men in their seventies have prostate cancer diagnosed at autopsy, while only $5-10 \%$ are detectable in a screening setting during life ${ }^{8}$.

Most guidelines, still mention the traditional cut-off of $4 \mathrm{ng} / \mathrm{ml}$ as an indication for biopsy. An increase in the number of core samples per biopsy have been advocated, based on the observation that more cancers are diagnosed when more biopsies are taken. Cut-off values of PSA for biopsy have been reduced in some areas of the world due to the detection of significant cancers in the low PSA range ${ }^{9}$. The awareness of prostate cancer in the general population has been increased due to print and electronic media and also from the urologic profession $^{9}$. If the current trends of transmission of information about prostate cancer continue, the number of living men diagnosed with prostate cancer will increase even further ${ }^{11}$. Prostate cancer can often be found early by testing prostate-specific antigen (PSA) in a man's blood digital rectal exam (DRE). If the results of either one of these tests are abnormal, further testing is needed to see if there is a cancer. If prostate cancer is found as a result of screening with the PSA test or DRE, it will probably be at an earlier, more treatable stage than if no screening were done

Routine screening for prostate cancer in US since 1990, the prostate cancer death rate has dropped. But it is not 
clear that this reduced death rate is a direct result of screening. It could also be caused by other factors like improvements in treatment of prostate cancer.

This is clear that screening program may help to find many prostate cancers early, but there are problems connected to the prostate cancer screening tests used today. Both the PSA test and the DRE are not 100\% accurate. These tests can sometimes have abnormal results even when a man does not have cancer (falsepositive results). Normal results can also occur even when a man does have cancer (false-negative results). Equivocal and unclear test results can cause confusion and anxiety and require more expansive and invasive test. False-positive results can lead some men to have a prostate biopsy when they do not have cancer. The trans rectal US guided biopsy may be associated with small risks of pain, infection, and bleeding. A false-negative results can give some men a false sense of security even though they actually have prostate cancer.

More importantly even if screening detects a cancer, doctors often can't tell whether this cancer is truly dangerous or not. Detecting and treating all prostate cancers early might seem as if it would always be a good thing, but some prostate cancers grow so slowly that they would probably never cause problem, may be it would never have led to their death, or even caused any symptoms. These men may still be treated with either surgery or radiation, which may be associated with urinary, bowel, and or sexual side effects that may seriously affect a man's quality of life.

Some of the detected cases particularly elderly peoples are to be followed without being treated right away and enrolled in a watchful waiting or active surveillance program. Although this group of patients is not treated right away, they still need regular blood tests and prostate biopsies to determine the need for future treatment. The entire program is linked with risks of anxiety, pain, infection, and bleeding. A large number of studies are going on to see if prostate cancer screening is worthwhile to reduce the risk of death from prostate cancer. The most recent results from 2 large studies were not conclusive and conflicting, and didn't offer clear answers.

Results from one study done in the United States found that annual screening with PSA and DRE did detect more prostate cancers than in men not screened, but this screening did not lower the death rate from prostate cancer. A European study did find a lower risk of death from prostate cancer with PSA screening (done about once every 4 years), but the researchers estimated that about 1,050 men would need to be screened (and 37 treated) in order to prevent one death from prostate cancer. Neither of these studies has shown that PSA screening helps men live longer (lowers the overall death rate).

The American Cancer Society recommends that men thinking about prostate cancer screening should make informed decisions based on available information, discussion with their doctor, and their own views on the benefits and side effects of screening and treatment.

Screening men who are older or in poor health in order to find early prostate cancer is less likely to help them live longer. This is because most prostate cancers are slow growing, and men who are older or sicker are likely to die from other causes before their prostate cancer grows enough to cause problems. The young people particularly if the prostate cancer runs in their family and develop prostate cancer, it may shorten your life if it's not caught early.

Currently there is no unanimous opinion in the medical community regarding the benefits of prostate cancer screening. Those who advocate regular screening believe that finding and treating prostate cancer early offers men more treatment options with potentially fewer side effects. Who recommend against regular screening note that because most prostate cancers grow very slowly, the side effects of treatment would likely outweigh any benefit that might be derived from detecting the cancer at a stage when it is unlikely to cause problems.

In 2012, the U.S. Preventative Task Force recommended against the use of PSA screening for healthy men of all ages, stating that the harms of screening outweigh the benefits. In contrast, physician-led groups, such as the American Society of Clinical Oncology and the American Urological Association, maintain that PSA screening should be considered in the context of a man's life expectancy and other medical conditions. Most experts agree that there is no role for PSA screening for men expected to live less than 10 years. Ultimately, decisions about screening should be individualized based on a man's level of risk, overall health, and life 
expectancy, as well as his desire for eventual treatment if he is diagnosed with prostate cancer.

The American Urological Association (AUA) Foundation changed and updated it's screening recommendations on May 3, 2013. The PSA test remains an important tool in the diagnostic process. Men over 40 should discuss PSA screening with their physicians to determine if and when PSA screening is right for them, based on health and family history factors, they differ from those of the U.S. Preventive Services Task Force (USPSTF).

\section{Timing for screening of Prostate cancer}

Appropriate age to start screening is generally based on individual risk, with age 40 being a reasonable time to start screening for those at highest risk for example genetic predispositions or strong family histories of prostate cancer at a young age. For otherwise healthy men at high risk (positive family history or African American men), starting at age 40-45 is reasonable. Most guidelines recommend an initial PSA and DRE at age 40 , but others recommend starting at age 50 . When to stop screening is also controversial. Some groups propose 75 as a reasonable cut-off age. Other groups suggest this is an individual decision based on life expectancy and overall current health.

\section{Indication of Biopsy of prostate}

A prostate cancer screening may reveal results that prompt a doctor to recommend a biopsy. There are many other supplementary tests and considerations that can help a man who is undergoing screening decide if a biopsy is necessary, including: Lower vs. higher free PSA test, PSA velocity (rate of rise over time), PSA density (PSA per volume of prostate), Family history, Ethnicity, Prior biopsy findings, Abnormal digital rectal exam results and Different forms of PSA (i.e. bPSA, pro-PSA). In general, a lower free PSA (percentage) indicates a higher risk of finding cancer at biopsy, as does a higher PSA velocity and PSA density.

\section{Informed decision making, reduction of unnecessary prostate biopsies}

Unbiased and correct information regarding this procedure and its consequences should be offered to every man considering prostate testing. Validated information, well understandable text in local language should delivers the information in all countries around the world. It may be noted that it has been seen that such information reduces the number of men who initially wanted to be screened.

Men should also ask themselves if they are at risk for having prostate cancer, and if they would like to follow the procedure screening involving PSA testing, urologic investigations, prostate biopsies, and potential treatment. It is possible to provide risk assessments for every step of the screening procedure. This willful participation might support patients and doctors in their decision to follow or refrain from further steps, dependent on their interpretation of the risk calculated. At the time of cancer diagnosis it should be explained about the presence of prostate cancer and possible treatment should be discussed.

Much interest has been given to the increase of specificity of the biopsy procedure in the general population with serum markers like PSA isoforms and kallekreins in order to diminish the number of false negative biopsies ${ }^{12,13}$. Enhancing specificity always resulted in a reduction of sensitivity of prostate cancers. Based on autopsy incidence, the number of potentially detectable tumors is manifold the number of currently diagnosed cancers. It is therefore not the absolute number, but the number of clinically relevant cancers that is of interest ${ }^{14}$. The PSA isoforms and kallekreins should therefore ideally be related to the characteristics of the cancers detected.

To reduce over diagnosis in a screening setting, markers are needed that reduce the risk on a positive prostate biopsy, increasing the specificity of this procedure. Men from the age of forty, as well as their advising doctors, need instruments to reduce their doubts and anxiety of the potential presence of a prostate cancer. This, together with balanced information about the benefits and risks of the individual outcome of screening procedures, might induce a more selective and step-wise screening action. Risk assessment, incorporating the main determinants known for the presence of prostate cancer from the age of 50, such as age, family history, and micturition complaints, should form the base of an individual screening approach. Objective values of serum markers might enhance the accuracy of such of risk predictors. prostate-cancer-specific mortality, levels of over diagnosis may remain unacceptable for population-based screening. 


\section{Bangladesh Perspective}

Bangladesh is one of the small developing countries in south Asia. The prostate cancer prevalence is low compared to the developed world. But the prevalence of prostate cancer in Bangladeshi urban population is increasing as indicated by the increased number of patient are currently diagnosed with prostate cancer. In 1980s most of the prostate cancer was diagnosed as advanced cases and the treatment option was bilateral orchectomy. The scenario has been changed with time. Increased number of early and advanced cases of prostate cancer cased are detected in clinical practices. There may be various reasons for such a higher prevalence. The life expectancy of Bangladeshi population has been increased significantly; more peoples are health conscious and involved in health check program. As Serum PSA is included in the health check program they are referred to the urology OPD or Urologist consultation where they may be diagnosed with a prostate cancer. Urologist population is also increased in Bangladesh there are about 200 Urologist working in different hospitals throughout the country. An indirect screen is always taken place while elderly men presented to the general out patient department with lower urinary tract symptom, they are evaluated for their LUTS and a PSA test is included as a mandatory in their evaluation. Most urban dweller knows some information about Prostate cancer and its implication and it was observed that many times elderly men discuss their doctors about testing his PSA. Currently most of the district city general hospitals provide PSA Test. In hospital practice the early detection become possible through the PSA test and Digital rectal examination.

\section{Conclusions:}

It is still too early to say whether population-based prostate cancer screening is a useful tool with regard to cancer mortality. We must wait until the results of ongoing prostate cancer screening trials are available. Until then, routine population screening may not be encouraged. Those men who do want a PSA test should participate in carefully designed, balanced program. Various efforts are performed to find new markers in the proteome and genome of blood and urine. Based on large and longitudinal serum collections of men diagnosed with prostate cancer in screening settings, the EC-sponsored P-MARK consortium evaluates candidate markers as prognostic tools ${ }^{15}$.

Until alternative screening tools are found, PSA will continue to be used, and over diagnosis will remain an unavoidable drawback of prostate cancer screening. The current challenge is to ensure that, over diagnosis should not result in overtreatment. To this end, research efforts presently focus on clarifying which cancers can be managed through active surveillance.

\section{References:}

1. de Koning HJ, Auvinen A, Berenguer Sanchez A et al (2002) Large-scale randomized prostate cancer screening trials: program performances in the European randomized screening for prostate cancer trial and the prostate, lung, colorectal and ovary cancer trial. Int J Cancer 97(2):237-244.

2. Lowe JB, Ball J, Lynch BM et al (2004) Acceptability and feasibility of a community-based screening programme for melanoma in Australia. Health Promot Int 19(4):437-444.

3. Van Iersel CA, de Koning HJ, Draisma G et al (2006) Riskbased selection from the general population in a screening trial: selection criteria, recruitment and power for the DutchBelgian randomised lung cancer multi-slice CT screening trial (NELSON). Int J Cancer (in press).

4. Henschke CI, Yankelevitz DF, Libby DM, Pasmantier MW, Smith JP, Miettinen OS (2006). Survival of patients with stage I lung cancer detected on CT screening. N Engl J Med 355(17):1763-1771.

5. O’Brien B, Nichaman L, Browne JE, Levin DL, Prorok PC, Gohagan JK (2000) Prostate, lung, colorectal and ovarian cancer screening trial project team. coordination and management of a large multicenter screening trial: the prostate, lung, colorectal and ovarian (PLCO) cancer screening trial. Control Clin Trials 21(Suppl 6):310S-328S.

6. Catalona WJ, Smith DS, Ratliff TL et al (1991) Measurement of prostate-specific antigen in serum as a screening test for prostate cancer. N Engl J Med 324(17):1156-1161. [PubMed]

7. Parkin DM, Bray F, Ferlay J, Pisani P (2005) Global cancer statistics, 2002. CA Cancer J Clin 55(2):74-108.

8. Sakr WA, Grignon DJ, Crissman JD et al (1994) High grade prostatic intraepithelial neoplasia (HGPIN) and prostatic adenocarcinoma between the ages of 20-69: an autopsy study of 249 cases. In Vivo 8(3):439-443.

9. Nadler RB, Loeb S, Roehl KA, Antenor JA, Eggener S, Catalona WJ (2005) Use of $2.6 \mathrm{ng} / \mathrm{ml}$ prostate specific antigen 
prompt for biopsy in men older than 60 years. J Urol 174(6):2154-2157. [PubMed]

10. Aus G, Abbou CC, Bolla M et al (2005) EAU guidelines on prostate cancer. Eur Urol 48(4):546-551.

11. Welch HG, Schwartz LM, Woloshin S (2005) Prostate-specific antigen levels in the United States: implications of various definitions for abnormal. J Natl Cancer Inst 97(15):1132-1137.

12. Bangma $\mathrm{CH}$, Rietbergen JB, Kranse R, Blijenberg BG, Petterson K, Schroder FH (1997) The free-to-total prostate specific antigen ratio improves the specificity of prostate specific antigen in screening for prostate cancer in the general population. J Urol 157(6):2191-2196.

13. Raaijmakers R, de Vries SH, Blijenberg BG, Wildhagen MF, Postma R, Bangma CH, Darte C, Schröder FH (2007) HK2 and free PSA, a powerful prognostic combination in predicting minimal prostate cancer in screen-detected men within the PSA range 4-10 ng/mL. Eur Urol (submitted).

14. Yurdakul G, Bangma CH, Blijenberg BG, van Zelst BD, Wildhagen MF, van der Kwast TH, Schroder FH (2002) Different PSA assays lead to detection of prostate cancers with identical histological features. Eur Urol 42(2):154-158.

15. Van Gils MPMQ, Stenman UH, Schalken JA, Schröder FH, Luider TM, Lilja H, Bjartell A, Hemdy FC, Petterson KSI, Bischoff R, Takalo H, Nilsson O, Mulders PFA, Bangma CH (2005) Innovations in serum and urine markers in prostate cancer.Current European research in the P-Mark project. Eur Urol 48:1031-1041. 